CPTH-S377.0995

LA-UR-95-3420

hep-th/9509169

September 24, 1995

\title{
PHYSICAL STATES OF THE QUANTUM CONFORMAL FACTOR
}

\author{
Ignatios Antoniadis \\ Centre de Physique Théorique ${ }^{\dagger}$ \\ Ecole Polytechnique \\ 91128 Palaiseau, France \\ Pawel O. Mazur \\ Dept. of Physics and Astronomy \\ University of South Carolina \\ Columbia, SC 29208 USA \\ and \\ Emil Mottola \\ Theoretical Division, T-8 \\ Mail Stop B285 \\ Los Alamos National Laboratory \\ Los Alamos, NM 87545 USA
}

\begin{abstract}
The conformal factor of the spacetime metric becomes dynamical due to the trace anomaly of matter fields. Its dynamics is described by an effective action which we quantize by canonical methods on the Einstein universe $R \times S^{3}$. We find an infinite tower of discrete states which satisfy the constraints of quantum diffeomorphism invariance. These physical states are in one-to-one correspondence with operators constructed by integrating integer powers of the Ricci scalar.
\end{abstract}

$\dagger$ Laboratoire Propre du CNRS UPR A.0014 


\section{Introduction}

It is virtually certain that at the ultrashort Planck scale a theory of gravitational interactions requires a framework quite different from the familiar classical metric description of spacetime. At larger distance scales the metric becomes a useful variable and gravity should be described by an effective low energy field theory. The common assumption is that this effective field theory at large distance scales must be the Einstein theory. This is a reasonable supposition if one considers gravitational actions composed of local curvature invariants. Then, the Einstein-Hilbert action is the unique invariant containing no more than two derivatives of the spacetime metric and would be expected to dominate low energy gravitational physics. In recent works we have advanced the proposition that on the contrary, at cosmological distance scales quantum fluctuations of the metric can be important and modify drastically the classical metric description of general relativity [1].

This radical proposal stems from observations about the quantum trace anomaly of conformally coupled matter fields in an arbitrary curved background, and the effective non-local action it generates for gravitational interactions. Although the trace anomaly itself is a sum of local terms which are fourth order in curvature invariants, the anomalous trace can be described by a coordinate invariant effective action which is necessarily non-local in the full metric. Because of this non-locality the trace anomaly has consequences for long distance physics which may be quite different from that expected in the classical Einstein theory, and in particular for cosmological spacetimes. Whatever else a full quantum theory of gravity at the Planck scale entails, the trace anomaly due to massless fields remains, and its effects at large distance scales must be considered.

The trace anomaly originates from a conflict between coordinate invariance and scale invariance at the quantum level. This conflict is interesting because after all, a 
global dilation or scale transformation is just a particular coordinate transformation of conformally flat backgrounds (such as the Friedman-Robertson-Walker spacetime of classical cosmology). Why should quantum physics violate this particular coordinate invariance but no other? Does this partial breakdown of coordinate invariance survive in the full quantum theory? How is this global scale symmetry violation related to the infrared behavior of gravity at very large distance scales?

These questions can be addressed in the effective theory of the conformal factor which we have introduced and studied in several recent papers [1,2]. This paper extends and deepens that study by a detailed analysis of its canonical quantization and physical state space.

In order to isolate the effects of the trace anomaly in the conformal sector of gravity and study its infrared behavior, we introduce the conformal parameterization (or gauge),

$$
g_{a b}(x)=e^{2 \sigma(x)} \bar{g}_{a b}(x),
$$

in terms of which the $\sigma$-dependence of the anomaly induced action becomes local, as in two dimensions [3]. When this local $\sigma$ action is added to the classical action the total trace anomaly vanishes and scale invariance is restored in the quantum theory [2].

In the classical Einstein theory the trace part of the metric is constrained in terms of the matter sources and cannot fluctuate. In the effective action for $\sigma$ compelled by the trace anomaly this is not the case, and this new degree of freedom in the scalar sector is the source of a radical departure from the classical theory. The fluctuations of $\sigma$ induced by the trace anomaly become relevant in curved spacetimes at distance scales of the order of the curvature or greater. This may be seen, for example, by computing the graviton propagator in de Sitter spacetime, whose logarithmic growth at large spacelike separations leads to infrared divergences [4]. These infrared divergences in gravitational perturbation theory around cosmological background spacetimes are 
the signal of a departure from the classical Einstein theory, and the need for renormalization of gravity in the far infrared. Since the conformal anomaly records the effects of all massless fields (including gravitons) we have argued that the renormalization and infrared fixed point of gravity is controlled by the effective action for $\sigma$ ("infrared conformal dominance"). The effective $\sigma$ theory exhibits some remarkable properties. It is ultraviolet renormalizable and possesses a non-trivial infrared stable fixed point. This fixed point describes a scale invariant phase of quantum gravity which is characterized by certain anomalous scaling relations [1].

It is worthwhile to bear in mind that in $D=2$ dimensional gravity the situation is similar. There are no degrees of freedom in the Einstein action, which is a topological invariant in $D=2$. However, the trace anomaly of matter fields induces the PolyakovLiouville action for $\sigma$ which causes the metric to fluctuate [3]. This one new scalar degree of freedom is reflected in the value of the central charge which is shifted by one unit (from $c_{m}-26$ to $c_{m}-25$ ). The fluctuations of $\sigma$ modify the theory in a radical way through gravitational dressing of matter fields for example, even at very large distances where one may have expected quantum gravitational effects to be small. Anomalous dimensions and scaling relations for primary fields and their correlation functions may be computed, and exhibit behavior qualitatively different from the classical theory in a fixed metric background [5]. In four dimensions there are in addition transverse excitations of the metric which make their own contribution to the trace anomaly. However, even in the presence of gravitons the effective action for $\sigma$ has a certain universal form, dictated by general covariance and the structure of the trace anomaly for massless fields. Whatever the dynamics of the transverse graviton excitations the importance of this effective action for $\sigma$ is that it contains new dynamics over and above the classical theory which cannot be ignored.

An important difference between $D=2$ and $D=4$ is that the effective action 
induced by the trace anomaly of matter fields is quartic in derivatives, and immediately raises the question of unitarity, which typically plagues theories with actions with more than two derivatives. The fourth order action contains both a positive and negative norm scalar at the perturbative level, and the negative norm scalar can lead to a breakdown of unitarity in the presence of interactions. However, $\sigma$ is not just a scalar field but a particular component of the metric (1.1). In a metric theory there are diffeomorphism constraints which eliminate some of the perturbative excitations. Can one still make sense of the fourth order $\sigma$ action as a quantum theory? What are its physical excitations? Answering these questions is the principal motivation of this paper. The answer to the first question turns out to be in the affirmative, and the determination of the physical states of the $\sigma$ theory which survive all the quantum diffeomorphism constraints and their physical interpretation is our main result.

Before entering into the physical state conditions in a fourth order theory it is worthwhile to recall that even the second order Einstein-Hilbert Lagrangian exhibits a negative norm scalar which leads to an unbounded Euclidean action if treated in a naive fashion. In the classical Einstein theory this field is non-propagating (except possibly for a finite number of modes in spacetimes with certain topologies). It can be eliminated from the theory by the constraints of coordinate invariance [6], which in a canonical framework are just the $T^{0 i}$ and $T^{00}$ components of the equations of motion. In the Einstein theory this may be understood by simple counting of degrees of freedom. One begins with a real, symmetric metric which contains ten degrees of freedom. Four of these are pure gauge, corresponding to the general four vector parameterizing infinitesimal diffeomorphisms. In a canonical framework the vanishing of the momenta corresponding to these four invariances lead to four secondary first class constraints, which reduce the number of true propagating degrees of freedom to $10-4-4=2$ transverse gravitons in the Einstein theory. The negative norm scalar 
$\sigma$ of the Einstein theory has dropped out, or more precisely, it is constrained and determined by the matter sources. When the trace anomaly is taken into account this counting of degrees of freedom is modified by the appearance of a quartic action for $\sigma$, which (since the classical action was already second order) has the effect of introducing one additional degree of freedom into the quantum theory in an analogous way to the Liouville theory in two dimensions, which shifts $c_{m}-26 \rightarrow c_{m}-25$. The question now becomes: what physical states does this additional degree of freedom contain over and above the gravitons of the low-energy Einstein theory?

In this paper we study the canonical quantization and the issue of unitarity of the quartic effective theory induced by the trace anomaly in four dimensions. We quantize the effective action of the $\sigma$ field in terms of canonical commutation relations of positive and negative metric in the Fock space of modes propagating in the spacetime background $R \times S^{3}$. Then, following the methods of the previous paper [7] (hereafter denoted as Paper I in this work) we compute the moments of the energy-momentum tensor deduced from the same effective $\sigma$ action and derive the constraints of spatial diffeomorphisms on the three sphere. We then apply the positive frequency part of these constraints to the Hilbert space of states in the theory, level by level, and determine the physically allowed states in the Fock space which satisfy all the constraints of spatial diffeomorphism invariance. Lastly, we apply the quantum Hamiltonian constraint with the finite shift determined in Paper I. Although there is no propagating local degree of freedom (negative metric or otherwise) we find an infinite tower of discrete states which satisfy all the constraints. It should be emphasized that all these invariant states in the pure $\sigma$ theory are present independently of the ordinary local spin two excitations of the metric.

Depending on the value of the anomaly coefficient we can distinguish two cases. In the first case the Hamiltonian constraint is satisfied with a real momentum eigenvalue 
of the zero mode of $\sigma$ in this background. In this case, corresponding to $c_{m}>25$ in $D=2$, we can carry out the analysis of unitarity and show that the discrete physical states have manifestly positive norm. In the second case the Hamiltonian constraint is satisfied with an imaginary momentum eigenvalue, corresponding to $c_{m}<1$ in two dimensions. In this case the physical states are non-normalizable but instead correspond to operators of scalar observables. In fact, in the semiclassical limit, they are created by integer powers of the Ricci scalar operator integrated over spacetime. Because of the trace anomaly, the Ricci scalar is not constrained to be a constant by the equations of motion in the quantum theory and powers of this quantity correspond explicitly to different allowed physical states.

The outline of the paper is as follows. In Section 2 we introduce the canonical quantization of the $\sigma$ field in terms of Fock space oscillators with both positive and negative metric on $R \times S^{3}$. The advantages of quantizing in the background Einstein universe and the general framework are laid out in detail in paper I, the Appendix of which contains many relations involving the spherical harmonic functions which we make extensive use of in this paper as well. From the energy-momentum tensor for the $\sigma$ field we derive detailed expressions for the moments of its $T^{0 i}$ components with the volume non-preserving and volume preserving harmonics on $S^{3}$. In Section 3 we apply the positive frequency part of these moments to the possible states level by level in the Fock space. A particular linear combination of Fock space operators raised to the $n^{\text {th }}$ power yields a physical state at each even level $2 n$, which survives the application of all the constraints. In Section 4, the correspondence of the tower of allowed physical states with operators of well-defined scaling dimension in four dimensions is presented. We also repeat the analysis of physical states with the addition of conformally coupled scalar matter, and show that the interpretation is robust under the addition of matter fields. Finally, Section 5 contains our main conclusions and a discussion of the results. 


\section{Quantization of $\sigma$}

In paper I we have presented the general framework of canonical quantization on $R \times$ $S^{3}$, the diffeomorphism constraints and the correct subtraction of the Hamiltonian $H$ determined by the ghost system. This discussion applies to any theory with general coordinate invariance and zero stress-tensor trace. We examine now the particular case of the theory of the conformal factor induced by the trace anomaly. The induced effective action for $\sigma$ in spacetimes of Lorentzian signature is [1]

$$
\Gamma=-\frac{Q^{2}}{(4 \pi)^{2}} \int d^{4} x \sqrt{-g}\left[\sigma \Delta_{4} \sigma+\frac{1}{2}\left(G-\frac{2}{3} \square R\right) \sigma\right]
$$

where the Weyl covariant fourth order operator is

$$
\begin{aligned}
\Delta_{4} & \equiv \square^{2}+2 R^{a b} \nabla_{a} \nabla_{b}-\frac{2}{3} R \square+\frac{1}{3}\left(\nabla^{a} R\right) \nabla_{a} \\
& =\square^{2}+4 \partial_{t}^{2} \quad \text { on } \quad R \times S^{3}
\end{aligned}
$$

with unit radius, so that $R_{0 a}=0, R_{i j}=2 \delta_{i j}, R=6$. The parameter $Q^{2}$ is proportional to the coefficient of the Gauss-Bonnet term $G=R_{a b c d} R^{a b c d}-4 R_{a b} R^{a b}+R^{2}$ in the general form of the trace anomaly for conformal fields, and therefore depends on the matter content of the theory coupled to $\sigma$. Although it is known that $Q^{2}>0$ for all free matter fields we treat $Q^{2}$ as a free parameter in the following development.

The equation of motion for $\sigma$ derived from the action $\Gamma$ is

$$
\begin{aligned}
\Delta_{4} \sigma & =-\frac{1}{4}\left(G-\frac{2}{3} \square R\right) \\
& =0 \quad \text { on } \quad R \times S^{3},
\end{aligned}
$$

because $G=\square R=0$ on $R \times S^{3}$. Hence, $\sigma$ is a free field which may be expanded in functions of the form, $\exp (-i \omega t) Y_{J M}$ with $Y_{J M}$ the scalar harmonics of the 3 -sphere, for which the operator $\Delta_{4}$ factorizes as

$$
\left.\Delta_{4}\left\{\exp (-i \omega t) Y_{J M}\right\}=\left(\omega^{2}-(2 J)^{2}\right)\right)\left(\omega^{2}-(2 J+2)^{2}\right) \exp (-i \omega t) Y_{J M} .
$$


The sourcefree wave equation and simple factorization of the fourth order operator $\Delta_{4}$ are important simplifications that hold on the Einstein universe $R \times S^{3}$. The two factors correspond exactly to two independent free fields which we shall designate $\sigma_{1}$ and $\sigma_{2}$, respectively, each obeying second order wave equations. Each of these fields may be expanded in terms of canonical creation and destruction operators:

$$
\begin{aligned}
\sigma_{1} & =\frac{\pi}{Q} \sum_{J M}^{\prime} \frac{1}{\sqrt{2 J(2 J+1)}}\left(e^{-2 i J t} Y_{J M} a_{J M}+e^{2 i J t} Y_{J M}^{*} a_{J M}^{\dagger}\right) \\
\sigma_{2} & =\frac{\pi}{Q} \sum_{J M} \frac{1}{\sqrt{2(2 J+1)(J+1)}}\left(e^{-2 i(J+1) t} Y_{J M} b_{J M}+e^{2 i(J+1) t} Y_{J M}^{*} b_{J M}^{\dagger}\right),
\end{aligned}
$$

where the prime denotes the omission of the $J=0$ mode from the first oscillator sum. Its place is taken by the zero mode with linear time dependence, i.e.

$$
\sigma=\sigma_{0}+\sigma_{1}+\sigma_{2}, \quad \text { with } \quad \sigma_{0}=\frac{1}{Q}(\hat{q}+\hat{p} t) .
$$

The normalization of the modes in (2.5) is fixed by the normalization of the action (2.1). If the oscillator creation and destruction operators are normalized in the usual way,

$$
\begin{aligned}
& {\left[a_{J_{1} M_{1}}, a_{J_{2} M_{2}}^{\dagger}\right]=\delta_{J_{1} J_{2}} \delta_{M_{1} M_{2}}} \\
& {\left[b_{J_{1} M_{1}}, b_{J_{2} M_{2}}^{\dagger}\right]=-\delta_{J_{1} J_{2}} \delta_{M_{1} M_{2}} \quad \text { for } \quad Q^{2}>0,}
\end{aligned}
$$

then we find that the Feynman function of the full $\sigma$ field satisfies the correctly normalized inhomogeneous wave equation,

$$
i \Delta_{4}\left\langle T \sigma(x) \sigma\left(x^{\prime}\right)\right\rangle=\frac{8 \pi^{2}}{Q^{2}} \delta^{4}\left(x, x^{\prime}\right)
$$

provided that the zero mode coordinate and momentum obey

$$
[\hat{p}, \hat{q}]=-i
$$

Because the action $\Gamma$ is fourth order in derivatives, there are necessarily both positive and negative norm states in the Fock space generated by the $a^{\dagger}$ and $b^{\dagger}$, and 
the classical energy-momentum of the theory is not positive. The minus sign in the second of the commutation relations (2.7) signifies that $\sigma_{2}$ is quantized with negative metric if $Q^{2}>0$ while $\sigma_{1}$ is a positive metric field. Conversely, if $Q^{2}<0$ then $\sigma_{1}$ should be quantized with negative metric, while $\sigma_{2}$ becomes the positive metric field, and the signs in (2.7) and (2.8) should be reversed. Hence, in either case there are certainly negative norm states in the Fock space prior to the imposition of the diffeomorphism constraints. In order to find these constraints we need the energy-momentum tensor corresponding to the action $\Gamma$. This normal ordered energy-momentum tensor is given by

$$
\begin{aligned}
-\frac{(4 \pi)^{2}}{Q^{2}} T^{a b} & =: 2 \square\left(\nabla^{a} \sigma \nabla^{b} \sigma\right)+\frac{4}{3} \nabla^{a} \nabla^{b}\left(\nabla^{c} \sigma \nabla_{c} \sigma\right)+4 \nabla^{(a} \sigma \nabla^{b)} \square \sigma-4 \nabla_{c} \nabla^{(a}\left(\nabla^{b)} \sigma \nabla^{c} \sigma\right) \\
& -\frac{2}{3} \nabla^{a} \nabla^{b} \square \sigma+8 R_{c}^{(a} \nabla^{b)} \sigma \nabla^{c} \sigma-\frac{4}{3} R^{a b}\left(\nabla^{c} \sigma \nabla_{c} \sigma\right)-\frac{4}{3} R \nabla^{a} \sigma \nabla^{b} \sigma \\
& +\frac{2}{3} R^{a b} \square \sigma+\frac{2}{3} \sigma \nabla^{a} \nabla^{b} R+2 \nabla^{a} \nabla^{b}(R \sigma)+4 \square\left(R^{a b} \sigma\right)-8 \nabla^{c} \nabla^{(a}\left(R_{c}^{b)} \sigma\right) \\
& +4 \nabla_{c} \nabla_{d}\left(R^{c(a b) d} \sigma\right)-\frac{2}{3} \nabla^{(a}\left(\sigma \nabla^{b)} R\right)-2 R_{c d e}^{a} R^{b c d e} \sigma+8 R^{a c} R_{c}^{b} \sigma-2 R^{a b} R \sigma \\
& +g^{a b}\left\{(\square \sigma)^{2}-\frac{1}{3} \square\left(\nabla^{c} \sigma \nabla_{c} \sigma\right)-2 R^{c d} \nabla_{c} \sigma \nabla_{d} \sigma+\frac{2}{3} R\left(\nabla^{c} \sigma \nabla_{c} \sigma\right)+\frac{2}{3} \square^{2} \sigma\right. \\
& \left.-2 \square(R \sigma)+4 \nabla^{c} \nabla^{d}\left(R_{c d} \sigma\right)+\frac{1}{3} \nabla^{c}\left(\sigma \nabla_{c} R\right)+\frac{1}{2}\left(G-\frac{2}{3} \square R\right) \sigma\right\}:
\end{aligned}
$$

Its trace is simply proportional to the $\sigma$ equation of motion,

$$
T_{a}^{a}=-2 \frac{Q^{2}}{(4 \pi)^{2}} \Delta_{4} \sigma=\frac{Q^{2}}{32 \pi^{2}}\left(G-\frac{2}{3} \square R\right)=0
$$

which vanishes by (2.3). This is no accident, of course, but is the result of the total anomaly cancellation in the $\sigma$ theory. Just as in two dimensions the "classical" trace from the linear term in the action $\Gamma$ is opposite in sign from the trace anomaly of matter fields which gave rise to the effective $\sigma$ action (2.1) in the first place. Hence the trace of the $\sigma$ energy-momentum tensor is precisely equal to minus the $\sigma$ variation of the action (2.1) which vanishes on $R \times S^{3}$. 
On $R \times S^{3}$ the energy density may be written in the form,

$$
\begin{aligned}
-\left.\frac{(4 \pi)^{2}}{Q^{2}} T^{00}[\sigma]\right|_{R \times S^{3}}= & : 2 \dot{\sigma} \square \dot{\sigma}-2 \ddot{\sigma} \square \sigma-\ddot{\sigma} \triangle \sigma-(\square \sigma)^{2}+\sigma \triangle \ddot{\sigma}-4 \dot{\sigma}^{2} \\
& +\triangle\left(\frac{2}{3} \dot{\sigma}^{2}-\sigma \ddot{\sigma}+\frac{1}{6} \triangle \sigma^{2}-\frac{1}{3} \sigma \triangle \sigma-\frac{2}{3} \square \sigma+4 \sigma\right):,
\end{aligned}
$$

where $\triangle \equiv \nabla^{i} \nabla_{i}$ is the Laplacian operator on $S^{3}$. The generator of the spatial diffeomorphism constraints is

$$
\begin{gathered}
-\left.\frac{(4 \pi)^{2}}{Q^{2}} T^{0 i}[\sigma]\right|_{R \times S^{3}}=:-2 \dot{\sigma} \nabla^{i} \square \sigma-2(\square \dot{\sigma})\left(\nabla^{i} \sigma\right)+2 \nabla^{i} \nabla^{c}\left(\dot{\sigma} \nabla_{c} \sigma\right)+2 \partial_{t} \nabla^{c}\left(\nabla^{i} \sigma \nabla_{c} \sigma\right) \\
-2 \square\left(\dot{\sigma} \nabla_{i} \sigma\right)-\frac{4}{3} \partial_{t} \nabla^{i}\left(\nabla^{c} \sigma \nabla_{c} \sigma\right)-4 \nabla^{i} \dot{\sigma}+\frac{2}{3} \nabla^{i} \square \dot{\sigma}+4 \dot{\sigma} \nabla^{i} \sigma: .
\end{gathered}
$$

Substituting the mode expansion (2.5) into (2.11) and forming the moments of the volume non-preserving diffeomorphisms with positive frequency part,

$$
\dot{T}_{J M}^{(+)} \equiv-i \sqrt{V} \int_{S^{3}} d \Omega\left(\nabla_{i} Y_{J M}^{*}\right) T^{0 i(+)}=\sqrt{V} \int_{S^{3}} d \Omega\left(\nabla_{i} Y_{J M}^{*}\right) \partial_{t} T^{00(+)}
$$

yields the following somewhat formidable expression for the moments:

$$
\begin{aligned}
& \left.\dot{T}_{J M}^{(+)}\right|_{t=0}=-\frac{2 J}{3} \sqrt{\frac{J}{2 J+1}}(J+1)(2 J+3)(Q-i \hat{p}) a_{J M} \\
& \quad+\frac{2 J}{3} \sqrt{\frac{J+1}{2 J+1}}(J+1)(2 J-1)(Q+i \hat{p}) b_{J M}+ \\
& \sum_{J_{1} M_{1}} \sum_{J_{2} M_{2}} \mathcal{C}_{J_{1} J_{2} M_{1} M_{2}}^{J M}\left\{\frac { ( J _ { 1 } + J _ { 2 } ) } { 2 \sqrt { J _ { 1 } J _ { 2 } } } a _ { J _ { 1 } M _ { 1 } } a _ { J _ { 2 } M _ { 2 } } \left\{\left(J_{1}-J_{2}\right)^{2}\left(J_{1}+J_{2}\right)\left(J_{1}+J_{2}+1\right)\right.\right. \\
& \left.\quad+\frac{J(J+1)}{3}\left[4 J_{1} J_{2}-4\left(J_{1}^{2}+J_{2}^{2}\right)-\left(J_{1}+J_{2}\right)+J(J+1)\right]\right\}_{J_{1}>0 ; J_{2}>0} \\
& +\frac{\left(J_{1}+J_{2}+1\right)}{\sqrt{J_{1}\left(J_{2}+1\right)}} a_{J_{1} M_{1}} b_{J_{2} M_{2}}\left\{4\left(J_{1}-J_{2}\right) J_{1}\left(J_{2}+1\right)+\left(J_{1}-J_{2}\right)\left(J_{1}+J_{2}+1\right)^{2}\left(J_{1}-J_{2}-1\right)\right. \\
& \left.\quad+\frac{J(J+1)}{3}\left[4\left(J_{1} J_{2}-J_{1}^{2}-J_{2}^{2}\right)+3 J_{1}-7 J_{2}-3+J(J+1)\right]\right\}_{J_{1}>0} \\
& +\frac{\left(J_{1}+J_{2}+2\right)}{2 \sqrt{\left(J_{1}+1\right)\left(J_{2}+1\right)}} b_{J_{1} M_{1}} b_{J_{2} M_{2}}\left\{\left(J_{1}-J_{2}\right)^{2}\left(J_{1}+J_{2}+1\right)\left(J_{1}+J_{2}+2\right)+\right. \\
& \left.\quad \frac{J(J+1)}{3}\left[4\left(J_{1} J_{2}-J_{1}^{2}-J_{2}^{2}\right)-3\left(J_{1}+J_{2}\right)-2+J(J+1)\right]\right\}
\end{aligned}
$$




$$
\begin{aligned}
+ & \frac{\left(J_{1}-J_{2}\right)}{\sqrt{J_{1} J_{2}}} \tilde{a}_{J_{2} M_{2}}^{\dagger} a_{J_{1} M_{1}}\left\{-4 J_{1} J_{2}\left(J_{1}+J_{2}+1\right)+\left(J_{1}-J_{2}\right)^{2}\left(J_{1}+J_{2}\right)\left(J_{1}+J_{2}+1\right)\right. \\
& \left.-\frac{J(J+1)}{3}\left[4\left(J_{1} J_{2}+J_{1}^{2}+J_{2}^{2}\right)+J_{1}+J_{2}-J(J+1)\right]\right\}_{J_{1}>J_{2}>0} \\
+ & \frac{\left(J_{1}-J_{2}-1\right)}{\sqrt{J_{1}\left(J_{2}+1\right)}} \tilde{b}_{J_{2} M_{2}}^{\dagger} a_{J_{1} M_{1}}\left\{\left(J_{1}-J_{2}\right)\left(J_{1}-J_{2}-1\right)\left(J_{1}+J_{2}+1\right)^{2}-\right. \\
& \left.\frac{J(J+1)}{3}\left[4\left(J_{1} J_{2}+J_{1}^{2}+J_{2}^{2}\right)+5 J_{1}+7 J_{2}+3-J(J+1)\right]\right\}_{J_{1}>J_{2}+1} \\
+ & \frac{\left(J_{1}-J_{2}+1\right)}{\sqrt{\left(J_{1}+1\right) J_{2}}} \tilde{a}_{J_{2} M_{2}}^{\dagger} b_{J_{1} M_{1}}\left\{\left(J_{1}-J_{2}\right)\left(J_{1}-J_{2}+1\right)\left(J_{1}+J_{2}+1\right)^{2}-\right. \\
& \left.\frac{J(J+1)}{3}\left[4\left(J_{1} J_{2}+J_{1}^{2}+J_{2}^{2}\right)+7 J_{1}+5 J_{2}+3-J(J+1)\right]\right\}_{J_{1}+1>J_{2}>0} \\
+ & \frac{\left(J_{1}-J_{2}\right)}{\sqrt{\left(J_{1}+1\right)\left(J_{2}+1\right)}} \tilde{b}_{J_{2} M_{2}}^{\dagger} b_{J_{1} M_{1}}\left\{\left(J_{1}+J_{2}+1\right)\left[4\left(J_{1}+1\right)\left(J_{2}+1\right)+\left(J_{1}-J_{2}\right)^{2}\left(J_{1}+J_{2}+2\right)\right]\right. \\
& \left.\left.-\frac{J(J+1)}{3}\left[4\left(J_{1} J_{2}+J_{1}^{2}+J_{2}^{2}\right)+11\left(J_{1}+J_{2}\right)+10-J(J+1)\right]\right\}_{J_{1}>J_{2}}\right\}
\end{aligned}
$$

Here the notation [7]

$$
\begin{aligned}
\tilde{a}_{J M} & \equiv \epsilon_{M} a_{J-M} \\
\tilde{b}_{J M} & \equiv \epsilon_{M} b_{J-M} \quad \text { with } \\
\epsilon_{M} & \equiv(-)^{m-m^{\prime}}
\end{aligned}
$$

has been used, and the symbol $\mathcal{C}$ is an $O(4)$ angular momentum coupling coefficient that may be expressed as a product of two ordinary $S U(2)$ Clebsch-Gordon coefficients $C_{J_{1} m_{1} J_{2} m_{2}}^{J m}$ via

$$
\begin{aligned}
\mathcal{C}_{J_{1} M_{1} J_{2} M_{2}}^{J M} & \equiv \frac{\sqrt{V}}{\sqrt{\left(2 J_{1}+1\right)\left(2 J_{2}+1\right)}} \int_{S^{3}} d \Omega Y_{J M}^{*} Y_{J_{1} M_{1}} Y_{J_{2} M_{2}} \\
& =\frac{1}{\sqrt{(2 J+1)}} C_{J_{1} m_{1} J_{2} m_{2}}^{J m} C_{J_{1} m_{1}^{\prime} J_{2} m_{2}^{\prime}}^{J m^{\prime}} .
\end{aligned}
$$

The moments of the volume preserving diffeomorphisms may be constructed by substituting the mode expansions (2.5) into (2.12) and computing

$$
X_{J \mathcal{M}}^{(+)} \equiv-\int_{S^{3}} d \Omega \mathcal{Y}_{i_{J \mathcal{M}}} T^{0 i(+)}
$$

where the $\mathcal{Y}_{J \mathcal{M}}^{i}$ are the transverse vector harmonics on $S^{3}$ as defined in paper I. In a 
calculation analogous to that leading to (2.13) we find

$$
\begin{aligned}
& \left.X_{J \mathcal{M}}^{(+)}\right|_{t=0}=\frac{-i}{4} \sum_{J_{1} M_{1}}^{\prime} \sum_{J_{2} M_{2}}^{\prime} \mathcal{K}_{J_{1} M_{1} J_{2} M_{2}}^{J M}\left\{\frac{\left(J_{1}-J_{2}\right)}{2 \sqrt{J_{1} J_{2}}} a_{J_{1} M_{1}} a_{J_{2} M_{2}}\left[4 J(J+1)-4\left(J_{1}+J_{2}\right)^{2}+1\right]\right. \\
& +\frac{1}{\sqrt{J_{1}\left(J_{2}+1\right)}} a_{J_{1} M_{1}} b_{J_{2} M_{2}}\left\{\left[4 J(J+1)-4\left(J_{1}+J_{2}+1\right)^{2}+1\right]\left(J_{1}-J_{2}-1\right)-16 J_{1}\left(J_{2}+1\right)\right\} \\
& \left.+\frac{\left(J_{1}-J_{2}\right)}{2 \sqrt{\left(J_{1}+1\right)\left(J_{2}+1\right)}} b_{J_{1} M_{1}} b_{J_{2} M_{2}}\left[4 J(J+1)-4\left(J_{1}+J_{2}+2\right)^{2}+1\right]\right\} \\
& +\frac{1}{\sqrt{J_{1} J_{2}}} \tilde{a}_{J_{2} M_{2}}^{\dagger} a_{J_{1} M_{1}}\left\{\left[4 J(J+1)-4\left(J_{1}-J_{2}\right)^{2}+1\right]\left(J_{1}+J_{2}\right)+16 J_{1} J_{2}\right\}_{J_{1} \geq J_{2}} \\
& +\frac{\left(J_{1}+J_{2}+1\right)}{\sqrt{J_{1}\left(J_{2}+1\right)}} \tilde{b}_{J_{2} M_{2}}^{\dagger} a_{J_{1} M_{1}}\left[4 J(J+1)-4\left(J_{1}-J_{2}-1\right)^{2}+1\right]_{J_{1} \geq J_{2}+1} \\
& +\frac{\left(J_{1}+J_{2}+1\right)}{\sqrt{\left(J_{1}+1\right) J_{2}}} \tilde{a}_{J_{2} M_{2}}^{\dagger} b_{J_{1} M_{1}}\left[4 J(J+1)-4\left(J_{1}-J_{2}+1\right)^{2}+1\right]_{J_{1}+1 \geq J_{2}} \\
& +\frac{1}{\sqrt{\left(J_{1}+1\right)\left(J_{2}+1\right)}} \tilde{b}_{J_{2} M_{2}}^{\dagger} b_{J_{1} M_{1}}\left\{\left[4 J(J+1)-4\left(J_{1}-J_{2}\right)^{2}+1\right]\left(J_{1}+J_{2}+2\right)\right. \\
& \left.\left.-16\left(J_{1}+1\right)\left(J_{2}+1\right)\right\}_{J_{1} \geq J_{2}}\right\}
\end{aligned}
$$

where the coupling coefficient with the transverse vector $O(4)$ spherical harmonic $\mathcal{Y}_{J \mathcal{M}}^{i}$ is defined by

$$
\mathcal{K}_{J_{1} M_{1} J_{2} M_{2}}^{J \mathcal{M}} \equiv \frac{1}{2 \sqrt{\left(2 J_{1}+1\right)\left(2 J_{2}+1\right)}} \int_{S^{3}} d \Omega \mathcal{Y}_{J \mathcal{M}}^{i} Y_{J_{1} M_{1}} \nabla_{i} Y_{J_{2} M_{2}}
$$

The lowest moments of the diffeomorphism generators are just the $S O(4,2)$ conformal group generators which now have an explicit realization in terms of the $\sigma$ field creation and destruction operators. The lowest non-trivial moment of the volume nonpreserving diffeomorphism generators is

$$
\begin{aligned}
K_{M}^{(+)} & =\left.\dot{T}_{\frac{1}{2} M}^{(+)}\right|_{t=0}=-(Q-i \hat{p}) a_{\frac{1}{2} M}+ \\
& \sqrt{2} \sum_{J M_{1} M_{2}} \mathcal{C}_{J+\frac{1}{2} M_{1} J M_{2}}^{\frac{1}{2} M}\left\{-2(J+1) \sqrt{J(2 J+1)} \tilde{a}_{J-M_{2}}^{\dagger} a_{\left(J+\frac{1}{2}\right) M_{1}}\right. \\
& \left.+(2 J+1) \sqrt{(J+1)(2 J+3)} \tilde{b}_{J-M_{2}}^{\dagger} a_{\left(J+\frac{1}{2}\right) M_{1}}-\sqrt{(J+1)(2 J+1)} \tilde{a}_{\left(J+\frac{1}{2}\right)-M_{1}}^{\dagger} b_{J M_{2}}\right\},
\end{aligned}
$$

which together with its Hermitian conjugate form the 8 special conformal generators of $R \times S^{3}$. 
The lowest moments of the volume preserving diffeomorphisms are precisely the 6 rotation generators of $S O(4)$,

$$
R_{M_{1} M_{2}}=-i \sum_{J M_{3} M_{4}} \int d \Omega \rho_{M_{1} M_{2}}^{i}\left(Y_{J M_{3}} \nabla_{i} Y_{J M_{4}}^{*}\right)\left(a_{J M_{4}}^{\dagger} a_{J M_{3}}-b_{J M_{4}}^{\dagger} b_{J M_{3}}\right) .
$$

With these expressions for the $8+6=14$ generators of the global conformal group in hand, it is straightforward to evaluate their commutators, and compare to the form expected from the classical Lie algebra of $S O(4,2)$. In particular, the quantum commutator

$$
\left[K_{M_{1}}^{(+)}, K_{M_{2}}^{(-)}\right]=2 \delta_{M_{1} M_{2}} H+2 R_{M_{1} M_{2}}
$$

yields the Hamiltonian operator $H$,

$$
H=\frac{1}{2} \hat{p}^{2}+\frac{1}{2} Q^{2}+2 \sum_{J M}\left\{J a_{J M}^{\dagger} a_{J M}-(J+1) b_{J M}^{\dagger} b_{J M}\right\}
$$

which differs from the spatial integral of the normal ordered $: T^{00}:$ by a well-defined c-number shift, $Q^{2} / 2$.

The additional $\frac{1}{2} Q^{2}$ contribution is the analog of that obtained in two dimensional quantum gravity $[3,8]$. In $D=2$ the Polyakov-Liouville effective action has the fully covariant non-local form [3],

$$
\frac{Q^{2}}{16 \pi} \int d^{2} x \sqrt{-g} \int d^{2} x^{\prime} \sqrt{-g^{\prime}} R(x) \square^{-1}\left(x, x^{\prime}\right) R\left(x^{\prime}\right)
$$

where

$$
Q^{2}=\frac{25-c_{m}}{6}, \quad D=2
$$

is fixed in terms of the matter central charge $c_{m}$, by the anomaly cancellation condition at the conformal fixed point. By calculating the stress tensor of the invariant action (2.23) we find an additional contribution to the energy density of the $\sigma$ action which on $R \times S^{1}$ results in a constant c-number shift in the Hamiltonian equal to $Q^{2} / 2$ on 
the cylinder. Alternatively, the shift is obtained just as readily from the $D=2$ global conformal algebra $S O(2,2) \cong S L(2, C)$ by computing the commutator $\left[L_{1}, L_{-1}\right]$ in the analog of (2.21).

In $D=4$ the fully covariant but non-local action is $[1,2]$,

$$
\frac{Q^{2}}{4 \pi^{2}} \int d^{4} x \sqrt{-g} \int d^{4} x^{\prime} \sqrt{-g^{\prime}}\left(G-\frac{2}{3} \square R\right)(x)\left(\Delta_{4}\right)^{-1}\left(x, x^{\prime}\right)\left(G-\frac{2}{3} \square R\right)\left(x^{\prime}\right) .
$$

When the conformal parameterization (1.1) is substituted into this non-local action we obtain (2.1) plus an additional $\sigma$ independent term of exactly the same form as (2.25) but evaluated in the background metric $\bar{g}_{a b}$. Varying this additional $\sigma$ independent action with respect to the background metric gives an additional background dependent term in the energy-momentum tensor of the form,

$$
\begin{aligned}
\bar{T}^{a b} & =\frac{\alpha}{(4 \pi)^{2}}\left[g^{a b}\left(\frac{1}{2} R^{2}-R_{c d} R^{c d}\right)+2 R^{a c} R_{c}^{b}-\frac{4}{3} R R^{a b}\right] \\
& +\frac{\beta}{(4 \pi)^{2}}\left[-\frac{1}{18} g^{a b}\left(R^{2}-4 \square R\right)+\frac{2}{9} R R^{a b}-\nabla^{a} \nabla^{b} R\right],
\end{aligned}
$$

with $\alpha=-\beta=-\frac{1}{2} Q^{2}$. On $R \times S^{3}$ with unit radius we obtain, in particular, the additional contribution

$$
\left.\bar{T}^{00}\right|_{R \times S^{3}}=\frac{Q^{2}}{4 \pi^{2}}
$$

Integrating this result over the unit $S^{3}$ with volume $V=2 \pi^{2}$ gives precisely the $\frac{1}{2} Q^{2}$ contribution to the total Hamiltonian in (2.22). Because $R \times S^{3}$ is a product space there is no shift contribution to $T^{0 i}$ in eq. (2.12).

It is also interesting to remark that the c-number shift in the Hamiltonian generator on the cylinder results from anomaly cancellation in the full quantum theory of matter plus $\sigma$ plus ghosts in $D=2$ and that this relation has a four dimensional analog as well. In fact the c-number shift is just the vacuum Casimir energy of all these fields on the manifold $R \times S^{1}$. The Casimir energy is determined by the trace anomaly coefficient for all fields [9], except the ghosts, and one finds

$$
\left.\left\langle 0\left|T^{(q u) 00}\right| 0\right\rangle\right|_{R \times S^{1}}=-\frac{1}{24 \pi}\left(c_{m}+1+(-26+24)\right)
$$


Now using the anomaly cancellation condition (2.24) and integrating over $S^{1}$ one finds for the total zero-point energy, $Q^{2} / 2-2$, where the shift -2 arises from the additional contribution of ghosts 24 in (2.28). That the ghost energy has a contribution in addition to its trace anomaly coefficient is a reflection of the fact that the ghost action arises from gauge-fixing and is therefore not fully coordinate invariant. The additional -2 shift can be computed for instance by evaluating the commutator $\left[L_{1}^{g h}, L_{-1}^{g h}\right]$ in the ghost sector [10].

In four dimensions the trace anomaly has the general form

$$
\left\langle 0\left|T_{a}^{(q u) a}\right| 0\right\rangle=b C^{2}+b^{\prime} G+\left(\frac{2}{3} b+b^{\prime \prime}\right) \square R
$$

where $C^{2}$ is the Weyl tensor squared. As in $D=2$, for conformally flat backgrounds (for which $C^{2}=0$ ), the expectation value of the full energy-momentum tensor of conformally invariant matter may be determined from the trace anomaly [9]. In $D=4$ the result is identical to $(2.26)$ with $\alpha=16 \pi^{2} b^{\prime}$ and $\beta=8 \pi^{2}\left(2 b+3 b^{\prime \prime}\right)$. Integrating this result over the unit $S^{3}$ we obtain for the additional c-number shift in the vacuum energy,

$$
\left.\int_{S^{3}} d \Omega\left\langle 0\left|T^{(q u) 00}\right| 0\right\rangle\right|_{R \times S^{3}}=2 \pi^{2}\left(-6 b^{\prime}+2 b+3 b^{\prime \prime}\right)
$$

Now, the conformal fixed point condition in four dimensions enforces a constraint on the anomaly coefficients $[1,2]$

$$
2 b^{\prime}+2 b+3 b^{\prime \prime}=0
$$

for the total of all fields including $\sigma$. This relation is a consequence of the condition that the $\beta$-function of the $R^{2}$ coupling in the effective action vanishes at the infrared fixed point. In addition, anomaly cancellation at the fixed point requires

$$
Q^{2}=-32 \pi^{2} b^{\prime}, \quad D=4
$$

which is the analog of (2.24) in $D=2$. Hence we find for the total Casimir energy

$$
\left.\int_{S^{3}} d \Omega\left\langle 0\left|T^{(q u) 00}\right| 0\right\rangle\right|_{R \times S^{3}}=\frac{1}{2} Q^{2}-4
$$


where the -4 is the additional contribution of the ghost computed in paper I and the analog of -2 in two dimensions.

The explicit form of the moments of the $\sigma$ energy-momentum tensor given by eqs. (2.13) and (2.17), together with the quantum Hamiltonian (2.22) and its finite shift are the elements we need to determine the physical states in the quantum $\sigma$ theory in the next section. 


\section{The Physical States}

The physical states of the pure $\sigma$ theory will be determined now by applying the physical state conditions [7],

$$
\begin{aligned}
\dot{T}_{J M}^{(+)}|p h y s\rangle & =0 \\
X_{J \mathcal{M}}^{(+)}|p h y s\rangle & =0 \\
H|p h y s\rangle & =4 \mid \text { phys }\rangle
\end{aligned}
$$

to all possible states in the Fock space. We note first that by considering only the energy-momentum tensor of the pure $\sigma$ action we shall be determining the physical spectrum of only the conformal sector of quantum gravity in the vacuum sector of any matter or transverse, graviton excitations we may wish to add in a more complete theory. We have nothing new to add about the inclusion of gravitons in the conformally invariant quantization scheme of this paper, and remark only that if included they will generate new physical states in the transverse sector over and above what we find in the pure $\sigma$ theory. However any states we find in the pure $\sigma$ theory will survive also in the full theory with the matter and/or transverse graviton sectors.

Before applying the quantum constraints let us make some observations about the purely classical $\sigma$ theory. Classically, one may use the mode expansions (2.5) and (2.6) with all quantities ordinary c-numbers. Because of the fourth order operator $\Delta_{4}$ there are two sets of wave modes parameterized by $a$ and $b$. There are no exponentially growing solutions because $\omega^{2} \geq 0$ for both sets of modes. However for $Q^{2}>0$ the energy density of all the $b$ modes is negative in (2.22). When all the classical diffeomorphism constraints are imposed $T^{0 i}=T^{00}=0$ these negative energy solutions are eliminated and there is no instability at the classical level (in the free $\sigma$ theory).

In the quantization scheme of the previous section we have traded negative energy states for negative norm states. Since the quantum constraints on the physical states (3.1) are weaker than the corresponding classical conditions, the elimination of unphys- 
ical negative norm states by the quantum diffeomorphism constraints is a non-trivial test that the quantum $\sigma$ theory must pass.

Let us begin by observing that the Fock vacuum $|0\rangle$ of the $a$ and $b$ oscillators is characterized by an additional quantum number which is the eigenvalue of the zero mode momentum operator

$$
\hat{p}|0, p\rangle=p|0, p\rangle
$$

The general state is constructed by operating with any number of $a^{\dagger}$ and $b^{\dagger}$ creation operators on a Fock vacuum of this kind. From the form of the Hamiltonian (2.22) we observe that each $a_{J M}^{\dagger}$ contributes $2 J$ to the energy while each $b_{J M}^{\dagger}$ contributes $2 J+2$, both of which are integers. Hence, we define the integer level $N$ of the general $\sigma$ state by

$$
H|N, p\rangle=\left(\frac{1}{2} p^{2}+\frac{1}{2} Q^{2}+N\right)|N, p\rangle
$$

Since terms with different $N$ cannot cancel, we can apply the $\dot{T}_{J M}^{(+)}$constraints to each $N$ level independently. The application of the volume non-preserving conditions in the pure $\sigma$ theory now proceeds in a way analogous to that described in paper I for a scalar matter field, level by level.

The ground state at level 0 clearly satisfies the volume non-preserving constraints,

$$
\dot{T}_{J M}^{(+)}|0, p\rangle=0
$$

and is therefore a candidate physical state. For $N=1$ the only possible state involves a linear combination of

$$
a_{\frac{1}{2} M}^{\dagger}|N=1, p\rangle
$$

Applying $\dot{T}_{\frac{1}{2} M}^{(+)}$to this is non-vanishing unless all the coefficients of this linear combination vanish identically. Hence there are no physical states at level one.

At level two, the general state is of the form,

$$
|2, p\rangle=\left(\alpha_{M_{1} M_{2}} a_{\frac{1}{2} M_{1}}^{\dagger} a_{\frac{1}{2} M_{2}}^{\dagger}+\beta b_{000}^{\dagger}+\gamma_{M} a_{1 M}^{\dagger}\right)|0, p\rangle .
$$


Applying $\dot{T}_{\frac{3}{2} M}^{(+)}$to this state gives a non-vanishing contribution unless

$$
\gamma_{M}=0
$$

identically. Applying $\dot{T}_{1 M}^{(+)}$gives no new constraint because the coefficient of $a_{\frac{1}{2}} a_{\frac{1}{2}}$ vanishes for $J=1$. Finally the $\dot{T}_{\frac{1}{2} M}^{(+)}$gives the non-trivial condition,

$$
\left[2(Q-i p) \alpha_{M M_{1}} a_{\frac{1}{2} M_{1}}^{\dagger}-\epsilon_{M} \beta a_{\frac{1}{2}-M}^{\dagger}\right]|0, p\rangle=0
$$

which is satisfied if

$$
\begin{aligned}
\text { i) } Q=i p \quad \text { and } \quad \beta=0, \quad \text { or } \\
\text { ii) } Q \neq i p \quad \text { and } \quad \alpha_{M_{1} M_{2}}=\frac{\beta}{2(Q-i p)} \epsilon_{M_{1}} \delta_{M_{1}-M_{2}} .
\end{aligned}
$$

We see that there is a non-trivial state surviving the application of the volume nonpreserving spatial diffeomorphism constraints at level 2 .

At level three the general state is created by a linear combination of the five operators, $a_{\frac{3}{2}}^{\dagger}, b_{\frac{1}{2}}^{\dagger}, a_{1}^{\dagger} a_{\frac{1}{2}}^{\dagger}, b_{0}^{\dagger} a_{\frac{1}{2}}^{\dagger}$, and $a_{\frac{1}{2}}^{\dagger} a_{\frac{1}{2}}^{\dagger} a_{\frac{1}{2}}^{\dagger}$. Application of $\dot{T}_{\frac{5}{2}}^{(+)}$forces the coefficient of the first term to vanish. Application of $\dot{T}_{\frac{3}{2}}^{(+)}$forces the coefficients of the next two terms to vanish, and application of $\dot{T}_{\frac{1}{2}}^{(+)}$forces the the coefficients of the last two terms to vanish. Hence there are no physical states at level three in the pure $\sigma$ theory.

At level four there are ten possible operators, namely

$$
a_{2}^{\dagger}, b_{1}^{\dagger}, a_{\frac{3}{2}}^{\dagger} a_{\frac{1}{2}}^{\dagger}, a_{1}^{\dagger} a_{1}^{\dagger}, a_{1}^{\dagger} b_{0}^{\dagger}, b_{\frac{1}{2}}^{\dagger} a_{\frac{1}{2}}^{\dagger}, a_{1}^{\dagger} a_{\frac{1}{2}}^{\dagger} a_{\frac{1}{2}}^{\dagger}, b_{0}^{\dagger} b_{0}^{\dagger}, b_{0}^{\dagger} a_{\frac{1}{2}}^{\dagger} a_{\frac{1}{2}}^{\dagger} \text {, and } a_{\frac{1}{2}}^{\dagger} a_{\frac{1}{2}}^{\dagger} a_{\frac{1}{2}}^{\dagger} a_{\frac{1}{2}}^{\dagger} \text {. }
$$

Applying $\dot{T}_{\frac{7}{2}}^{(+)}$to the general level 4 state forces the coefficient of the first term to vanish. $\dot{T}_{\frac{5}{2}}^{(+)}$eliminates the next two terms, $\dot{T}_{\frac{3}{2}}^{(+)}$eliminates the fourth and fifth operators, while $\dot{T}_{2}^{(+)}$and $\dot{T}_{1}^{(+)}$eliminate the sixth and seventh, respectively. We are left then with products of the same operators $b_{0}^{\dagger}$ and $a_{\frac{1}{2}}^{\dagger}$ which survived at level two. Applying the 
last constraint $\dot{T}_{\frac{1}{2}}^{(+)}$forces these operators to appear in precisely the same combination found at the lower level, squared, that is the allowed state at level four is of the form,

$$
|4, p\rangle=\left(\alpha_{M_{1} M_{2}} a_{\frac{1}{2} M_{1}}^{\dagger} a_{\frac{1}{2} M_{2}}+\beta b_{000}^{\dagger}\right)^{2}|0, p\rangle,
$$

where the same conditions (i) or (ii) of (3.7) must be satisfied.

This procedure can be continued indefinitely. At each level $N$, applying the $\dot{T}_{J M}^{(+)}$ constraints in the decreasing order $J=N-\frac{1}{2}, \ldots, \frac{1}{2}$ eliminates all the possible linear combinations of operators at that level except for $N$ even, where just that combination which appears at level 2 survives, raised to the power $N / 2$.

It is not difficult to check directly that the state

$$
|2 n, p\rangle=\left(\sum_{M_{1} M_{2}} \alpha_{\frac{1}{2} M_{1} M_{2}} a_{\frac{1}{2} M_{1}}^{\dagger} a_{\frac{1}{2} M_{2}}+\beta b_{000}^{\dagger}\right)^{n}|0, p\rangle
$$

indeed does satisfy all the volume non-preserving diffeomorphism constraints,

$$
\dot{T}_{J M}^{(+)}|2 n, p\rangle=0
$$

at general even level $N=2 n$, provided (i) or (ii) of (3.7) is satisfied. Taking careful note of the explicit form of $\dot{T}_{J M}^{(+)}(2.13)$ with the restrictions on $J_{1}$ and $J_{2}$ in the sum, one quickly observes that most of the bilinear operators annihilate the state $|2 n, p\rangle$ and therefore give no condition. Explicitly, the $a_{J_{1} M_{1}} a_{J_{2} M_{2}}$ terms annihilate the state unless $J_{1}=J_{2}=\frac{1}{2}$. The angular momentum coupling coefficient then vanishes unless $J=0$ or $J=1$, but in either of those cases the coefficient in curly brackets vanishes in (2.13) for this term. The $a_{J_{1} M_{1}} b_{J_{2} M_{2}}$ terms do not contribute unless $J_{1}=\frac{1}{2}$ and $J_{2}=0$ which implies $J=\frac{1}{2}$ but in this case the corresponding coefficient again vanishes. The $b_{J_{1} M_{1}} b_{J_{2} M_{2}}$ do not contribute since $J_{1}=J_{2}=0$ implies $J=0$ and the corresponding coefficient in curly brackets vanishes. The $\tilde{a}_{J_{2} M_{2}}^{\dagger} a_{J_{1} M_{1}}$ terms do not contribute unless $J_{1}=\frac{1}{2}$ but then $J_{2}$ cannot satisfy the restriction $\frac{1}{2}>J_{2}>0$. The $\tilde{b}_{J_{2} M_{2}}^{\dagger} a_{J_{1} M_{1}}$ terms do 
not contribute unless $J_{1}=\frac{1}{2}$ but then $J_{2}$ cannot satisfy the restriction $\frac{1}{2}>J_{2}+1$. The $\tilde{a}_{J_{2} M_{2}}^{\dagger} b_{J_{1} M_{1}}$ terms do not contribute unless $J_{1}=0$ but then $J_{2}=\frac{1}{2}$ and only the $J=\frac{1}{2}$ moment must be examined in detail. Finally, the $\tilde{b}_{J_{2} M_{2}}^{\dagger} b_{J_{1} M_{1}}$ terms do not contribute unless $J_{1}=0$ but the restriction $0>J_{2}$ cannot be satisfied. Since from the linear terms in (2.13) only the $a_{\frac{1}{2} M}$ term can contribute we have explicitly the only surviving terms at $J=\frac{1}{2}$,

$$
\dot{T}_{\frac{1}{2} M}^{(+)}|2 n, p\rangle=-\left((Q-i p) a_{\frac{1}{2} M}+\tilde{a}_{\frac{1}{2} M}^{\dagger} b_{00}\right)|2 n, p\rangle .
$$

Now one can verify that the commutator

$$
\left[(Q-i p) a_{\frac{1}{2} M}+\tilde{a}_{\frac{1}{2} M}^{\dagger} b_{00}, \alpha_{\frac{1}{2} M_{1} M_{2}} a_{\frac{1}{2} M_{1}}^{\dagger} a_{\frac{1}{2} M_{2}}^{\dagger}+\beta b_{00}^{\dagger}\right]=0
$$

if and only if one of the conditions (3.7) are satisfied. In that case the surviving operator above passes through the operator creating the state in (3.9) and annihilates the Fock vacuum. Hence the state $|2 n, p\rangle$ in (3.9) satisfies all the volume non-preserving spatial diffeomorphism constraints if the conditions (3.7) are satisfied.

By a similar exercise it is not difficult to check that the state $|2 n, p\rangle$ satisfies all the constraints of volume preserving spatial diffeomorphisms as well, viz.

$$
X_{J \mathcal{M}}^{(+)}|2 n, p\rangle=0 \text {. }
$$

Indeed, by inspecting the explicit form $(2.17)$ for $X_{J \mathcal{M}}^{(+)}$one quickly verifies that operating on this state the $a_{J_{1} M_{1}} a_{J_{2} M_{2}}$ terms do not contribute since $J_{1}=J_{2}=\frac{1}{2}$ has vanishing $J_{1}-J_{2}$. The $a_{J_{1} M_{1}} b_{J_{2} M_{2}}, b_{J_{1} M_{1}} b_{J_{2} M_{2}}, \tilde{a}_{J_{2} M_{2}}^{\dagger} b_{J_{1} M_{1}}$ and $\tilde{b}_{J_{2} M_{2}}^{\dagger} b_{J_{1} M_{1}}$ terms do not contribute unless $J_{2}=0$ which is not in the primed sum. Likewise, the $\tilde{b}_{J_{2} M_{2}}^{\dagger} a_{J_{1} M_{1}}$ term does not contribute unless $J_{1}=\frac{1}{2}$ but then the restriction $\frac{1}{2} \geq J_{2}+1$ excludes this term from the sum. Finally only the $\tilde{a}_{J_{2} M_{2}}^{\dagger} a_{J_{1} M_{1}}$ term remains with $J_{1}=\frac{1}{2}$. But then $J_{1} \geq J_{2}>0$ implies $J_{2}=\frac{1}{2}$ as well. Since in that case

$$
\rho_{M_{1} M_{2}}^{i}=i \frac{V}{4}\left(Y_{\frac{1}{2} M_{1}}^{*} \nabla^{i} Y_{\frac{1}{2} M_{2}}-Y_{\frac{1}{2} M_{2}} \nabla^{i} Y_{\frac{1}{2} M_{1}}^{*}\right)=\sqrt{\frac{2}{V}} \mathcal{Y}_{\frac{1}{2} \mathcal{M}}^{i}
$$


is just the lowest of transverse vector harmonics which are mutually orthogonal on $S^{3}$, the coupling coefficient $\mathcal{K}_{\frac{1}{2} M_{1} \frac{1}{2} M_{2}}^{J \mathcal{M}}$ vanishes unless $J=\frac{1}{2}$, so that only the $X_{\frac{1}{2} \mathcal{M}}^{(+)} \sim$ $R_{M_{1} M_{2}}$ moment condition needs to be verified in detail. Using the properties of $\rho^{i}$ given in the Appendix of paper I, this condition becomes

$$
R_{M_{1} M_{2}}|2 n, p\rangle=\left(a_{\frac{1}{2} M_{2}}^{\dagger} a_{\frac{1}{2} M_{1}}-\epsilon_{M_{1}} \epsilon_{M_{2}} a_{\frac{1}{2}-M_{1}}^{\dagger} a_{\frac{1}{2}-M_{2}}\right)|2 n, p\rangle=0
$$

which is satisfied if and only if the commutator

$$
\left[a_{\frac{1}{2} M_{2}}^{\dagger} a_{\frac{1}{2} M_{1}}-\epsilon_{M_{1}} \epsilon_{M_{2}} a_{\frac{1}{2}-M_{1}}^{\dagger} a_{\frac{1}{2}-M_{2}}, \sum_{M_{3} M_{4}} \alpha_{\frac{1}{2} M_{3} M_{4}} a_{\frac{1}{2} M_{3}}^{\dagger} a_{\frac{1}{2} M_{4}}^{\dagger}\right]=0,
$$

which is satisfied in turn if and only if

$$
\alpha_{\frac{1}{2} M_{1} M_{2}}=\alpha \epsilon_{M_{1}} \delta_{M_{1}-M_{2}}
$$

for some $\alpha$. This is just the condition that the state be $O(4)$ rotationally invariant. Recalling now the conditions (3.7) one finds that in both cases (i) and (ii) we may identify $\beta=2(Q-i p) \alpha$ and (3.15) is thereby satisfied. Hence, the state

$$
|2 n, p\rangle=\left(2(Q-i p) b_{00}^{\dagger}+\sum_{M} \epsilon_{M} a_{\frac{1}{2} M}^{\dagger} a_{\frac{1}{2}-M}^{\dagger}\right)^{n}|0, p\rangle
$$

satisfies this constraint of rotational invariance, and all the spatial diffeomorphism constraints.

The first remark to be made about this result is that it is interesting that any nontrivial physical states survive the application of the diffeomorphism constraints in the pure $\sigma$ theory. In two dimensions the corresponding procedure eliminates everything except the vacuum state. Clearly, the situation in four dimensions is much richer, since we find a physical state at every even level $N=2 n$.

It is also worthwhile to remark that the imposition of the full $T^{00}$ constraints of the classical theory would eliminate all of the physical states from the spectrum except 
the Fock vacuum. This is because $T^{00}$ contains a time-independent term for any $J$ proportional to

$$
\begin{aligned}
& \sum_{j M_{1} M_{2}} \mathcal{C}_{j M_{1} j-M_{2}}^{J M}\left\{\left[-4 j(2 j+1)-\frac{2}{3} J(J+1)(6 j+1)+\frac{1}{3 j} J^{2}(J+1)^{2}\right] a_{j M_{1}}^{\dagger} a_{j-M_{2}}\right. \\
& +\left[4(j+1)(2 j+1)-\frac{2}{3} J(J+1)(6 j+5)+\frac{1}{3(j+1)} J^{2}(J+1)^{2}\right] b_{j M_{1}}^{\dagger} b_{j M_{2}} \\
& \left.+\left[-4(j+1) J(J+1)+\frac{1}{3(j+1)} J^{2}(J+1)^{2}\right]\left(a_{j+1 M_{2}}^{\dagger} b_{j M_{1}}+b_{j M_{1}}^{\dagger} a_{j+1 M_{2}}\right)\right\} .
\end{aligned}
$$

Requiring this to vanish on the physical states would lead to the trivial solution at level zero alone. However, we know that imposing the $J=0$ moment of the naive $T^{00}$ constraint above is flatly inconsistent with the quantum Hamiltonian constraint with the correct subtraction determined in paper I.

Lastly, we consider the Hamiltonian constraint. From (3.3) we see that the Hamiltonian constraint determines the momentum eigenvalue $p_{2 n}$ at every even level $2 n$ to satisfy

$$
\frac{1}{2} p_{2 n}^{2}+\frac{1}{2} Q^{2}+2 n=4 .
$$

Hence the Hamiltonian physical state condition (3.17) cannot be satisfied for real $p$ if $Q^{2}>8$. This is similar to the situation in two dimensions for central charge $c_{m}<1$. In string theory this fact is interpreted to mean that $p$ is not actually a momentum at all but a timelike quantity more analogous to energy in the target space. Then we may define

$$
p_{2 n} \equiv i E_{2 n}
$$

and write the physical state condition in the form

$$
E_{2 n}=+\sqrt{Q^{2}-8+4 n},
$$

with $E_{2 n}$ real and positive. Although this continuation results in states which are not $\delta$-function normalizable, we recall from $c_{m} \leq 1$ in $2 \mathrm{D}$ gravity that they are created by operators with well-defined scaling properties. In this region of central charge the theory 
has a metric interpretation since these operators are matter primary fields dressed with normal ordered exponentials of the conformal factor with real exponents. In the next section we will see how this interpretation is generalized in four dimensions.

Since we have regarded $Q^{2}$ as a free parameter let us consider the situation when $Q^{2}<0$ which corresponds to $c_{m}>25$ in non-critical string theory. In this region the Hamiltonian condition (3.17) can be satisfied with real momenta, and the roles of the $a$ and $b$ oscillators are interchanged, the $a^{\dagger}$ oscillators now creating negative norm states. The physical states are still of the form (3.16) and manifestly have positive norm since only pairs of $a^{\dagger}$ operators appear together. This means that the negative norm states have completely disappeared from the spectrum after applying the diffeomorphism constraints. Therefore the pure $\sigma$ theory described by the fourth order action (2.1) is unitary for $Q^{2}<0$, and does not suffer from the problems plaguing local higher derivative theories of gravity in four dimensions. Since the addition of unitary matter and/or transverse graviton degrees of freedom should not disturb this feature of the $\sigma$ theory, we expect that the elimination of the negative norm states by the imposition of the diffeomorphism constraints will persist in the presence of these fields. This is indeed the case for free conformal scalar fields, as we verify in the end of the next section. The proper inclusion of transverse gravitons in this quantum $\sigma$ framework remains an open problem. 


\section{Operator Correspondence}

Since positive energy free matter fields give positive $Q^{2}$, which may well be larger than 8 [2], we return to this case and find the operators creating the physical states (3.16) and their geometric interpretation.

By analogy with the $D=2$ case we might guess that the general physical state of the pure $\sigma$ theory at level $2 n$ should correspond to dimension 4 operators of the form,

$$
\mathcal{O}_{4}^{(n)}=: F_{n}[\nabla \sigma] e^{\beta_{n} \sigma}:
$$

where $F_{n}$ is a polynomial containing derivatives up to order $2 n$ in $\sigma$ such that the full operator corresponds to a primary field with definite conformal weight equal to 4 . The conformal weight of a pure normal ordered exponential may be calculated in the canonical oscillator framework from the commutator,

$$
i\left[K_{M}^{( \pm)},: e^{y \sigma}:\right]=\kappa^{( \pm) a} \nabla_{a}: e^{y \sigma}:+\frac{w(y)}{4}: e^{y \sigma}: \nabla_{a} \kappa^{( \pm) a}
$$

upon using the Baker-Campbell-Haussdorf formula and the commutator (2.8) for the zero mode,

$$
\begin{aligned}
: e^{y \sigma}: & =e^{y \sigma^{(-)}} e^{y \sigma_{0}} e^{y \sigma^{(+)}} \\
& =e^{y \sigma^{(-)}} e^{\frac{\hat{q} y}{Q}} e^{\frac{y \hat{p} t}{Q}} e^{\frac{-i y^{2} t}{2 Q^{2}}} e^{y \sigma_{0}} e^{y \sigma^{(+)}},
\end{aligned}
$$

where $\sigma^{(+)}, \sigma^{(-)}, \sigma_{0}$ are the positive, negative and zero frequency components of $\sigma$. By inserting the mode expansions for $\sigma$, into the exponentials and comparing frequency level by frequency level with (4.2), we find that (4.2) is satisfied with

$$
w(y)=y-\frac{y^{2}}{2 Q^{2}} .
$$

The classical scaling dimension $y$ of the exponential is modified by a quantum correction which agrees with the covariant one-loop Feynman diagram calculation of ref. [1].

Hence, the pure exponential operator

$$
\int d^{4} x: e^{\beta_{0} \sigma}:
$$


is a diffeomorphism invariant scalar provided

$$
w\left(\beta_{0}\right)=\beta_{0}-\frac{\beta_{0}^{2}}{2 Q^{2}}=4 .
$$

The solution of this quadratic equation is

$$
\beta_{0}=Q\left(Q+i p_{0}\right)=Q\left(Q-E_{0}\right)
$$

with the same branch of the square root chosen as in (3.19) in order to reproduce the classical limit,

$$
\beta_{0} \rightarrow 4 \quad \text { as } \quad Q^{2} \rightarrow \infty
$$

In this limit we see that the normal ordered exponential operator with $\beta_{0}$ given by (4.7) is just the volume operator $\int d^{4} x \sqrt{-g}$ which is clearly diffeomorphism invariant.

By operating with this exponential on the Fock vacuum state $|\Omega\rangle$ annihilated by $H, K_{M}^{(+)}$, and $R_{M_{1} M_{2}}$ we obtain a state invariant under all spatial diffeomorphisms. Since

$$
H|\Omega\rangle=\frac{1}{2}\left(\hat{p}^{2}+Q^{2}\right)|\Omega\rangle=0
$$

the vacuum state $|\Omega\rangle$ must be an eigenstate of $\hat{p}$ with non-zero imaginary momentum. Choosing again the same sign of the square root as in (3.19),

$$
\hat{p}|\Omega\rangle=i Q|\Omega\rangle
$$

we find that the state constructed with the exponential operator is an eigenstate of $\hat{p}$ with eigenvalue $p_{0}=i E_{0}$,

$$
\begin{aligned}
\hat{p} \int d^{4} x: e^{\beta_{0} \sigma}:|\Omega\rangle & =\hat{p} \int d^{4} x: e^{\beta_{0} \sigma_{0}}:|\Omega\rangle \\
& =\left[\hat{p}, e^{\frac{\beta_{0} \hat{q}}{Q}}\right] e^{\frac{\beta_{0} p t}{Q}} e^{\frac{-i \beta_{0}^{2} t}{2 Q^{2}}}|\Omega\rangle+\int d^{4} x: e^{\beta_{0} \sigma}: \hat{p}|\Omega\rangle \\
& =\left(-\frac{i \beta_{0}}{Q}+i Q\right) \int d^{4} x: e^{\beta_{0} \sigma}:|\Omega\rangle \\
& =i \sqrt{Q^{2}-8} \int d^{4} x: e^{\beta_{0} \sigma}:|y\rangle .
\end{aligned}
$$


Finally, since the exponential operator has weight 4 and the Hamiltonian on $R \times S^{3}$ just measures the conformal weight [7], it follows that this state created by the exponential also satisfies the physical state Hamiltonian condition, $H|p h y s\rangle=4|p h y s\rangle$. Combining this with the eigenvalue of the momentum operator we conclude that the normal ordered exponential acting on the vacuum $|\Omega\rangle$ creates the physical state at level zero, i.e.

$$
\left|0, p_{0}\right\rangle \sim \int d^{4} x: e^{\beta_{0} \sigma}:|\Omega\rangle
$$

To proceed with the operator identification at higher levels we need to identify the proper primary operators of the form (4.1) that create the higher level physical states. Since $F_{n}$ contains up to $2 n$ derivatives of the $\sigma$ field, we would like to find that the conformal weight of the level $n$ operator be given by the formula,

$$
w_{n}=2 n+\beta_{n}-\frac{\beta_{n}^{2}}{2 Q^{2}}=4
$$

which would imply

$$
\beta_{n}=Q\left(Q+i p_{2 n}\right)=Q\left(Q-E_{2 n}\right)
$$

in analogy with relation (4.7) at level zero. However unlike in $D=2$ where simple normal ordering is sufficient to remove all divergences, in $D=4$ normal ordering operators of the form (4.1) only eliminates the leading (quartic) divergences of these composite operators, leaving behind, in general quadratic divergences as well as the logarithms wanted for conformal invariant behavior. Hence, we cannot expect normal ordered polynomial functions of $\sigma$ with a single exponential to have well-defined conformal dimensions or to create all the physical states for arbitrary finite $Q^{2}$ in four dimensions. The operator mixing problem requires the power of the operator product expansion of field theory and cannot be analyzed easily in the Fock space of oscillators of the present treatment. Indeed from the covariant $\sigma$ field theory analysis of the $\beta$-functions of the Einstein and cosmological couplings we know that such operator mixing does occur in 
the $\sigma$ theory [1]. Hence, we shall have to content ourselves here with an identification of operators with the physical states at $n>0$ only in the limit $Q^{2} \rightarrow \infty$ where this operator mixing problem disappears.

Since

$$
\beta_{n} \rightarrow 4-2 n \quad \text { as } \quad Q^{2} \rightarrow \infty
$$

it is not difficult to find the functions $F_{n}$ explicitly in this limit. Consider just the Ricci scalar of the full metric (1.1) raised to the power $n$ multiplied by $\sqrt{-g}$, i.e.

$$
\begin{aligned}
& : R^{n} \sqrt{-g}:=:\left[\bar{\square} \sigma+\bar{\nabla}^{a} \sigma \bar{\nabla}_{a} \sigma-\frac{\bar{R}}{6}\right]^{n} e^{(4-2 n) \sigma}: \\
& =:\left[-1-\dot{\sigma}_{o}^{2}+\bar{\square} \sigma_{q}-2 \dot{\sigma}_{0} \dot{\sigma}_{q}+\bar{\nabla}^{a} \sigma_{q} \bar{\nabla}_{a} \sigma_{q}\right]^{n} e^{(4-2 n) \sigma_{0}} e^{(4-2 n) \sigma_{q}}: \\
& =:\left[-1-\frac{p_{2 n}^{2}}{Q^{2}}-2 i\left(1-\frac{i p_{2 n}}{Q}\right) \dot{\sigma}_{2}+2 i\left(1+\frac{i p_{2 n}}{Q}\right) \dot{\sigma}_{1}+\bar{\nabla}^{a} \sigma_{q} \bar{\nabla}_{a} \sigma_{q}\right]^{n} e^{(4-2 n) \sigma_{0}} e^{(4-2 n) \sigma_{q}}: \\
& =:\left[-4 i \dot{\sigma}_{2}+\bar{\nabla}^{a} \sigma_{q} \bar{\nabla}_{a} \sigma_{q}+\ldots\right]^{n} e^{(4-2 n) \sigma_{0}} e^{(4-2 n) \sigma_{q}}:
\end{aligned}
$$

where $\sigma_{q}=\sigma_{1}+\sigma_{2}$ and the ellipsis contains terms with subleading $Q^{2}$ dependence. In the large $Q^{2}$ limit $\sigma_{q}$ is held fixed while $p_{2 n} \rightarrow i Q$. Substituting the mode expansions for $\sigma_{q}$, using the properties of the $J=\frac{1}{2}$ scalar harmonics catalogued in the Appendix of paper I, operating on the vacuum state, and integrating, one finds finally

$$
\int d^{4} x:\left[\frac{4}{Q} e^{2 i t} b_{00}^{\dagger}+\frac{1}{Q^{2}} e^{2 i t} \sum_{M} \epsilon_{M} a_{\frac{1}{2} M}^{\dagger} a_{\frac{1}{2}-M}^{\dagger}+\ldots\right]^{n} e^{(4-2 n) \sigma_{0}}:|\Omega\rangle .
$$

Frequencies higher than $\exp (2 i t)$ have been dropped in this last ellipsis since the time dependence of $\exp \left((4-2 n) \sigma_{0}\right)$ acting on the vacuum $|\Omega\rangle$ becomes just $\exp (4-2 n) i t$ in the large $Q^{2}$ limit, so that the total time dependence of the terms exhibited becomes $\exp (4 i t)$. It is this time dependence which is selected by the time integration as determined by the conformal transformation from flat space described in paper I. Hence in the classical $Q^{2} \rightarrow \infty$ limit the only surviving operator structure in (4.16) is just the 
linear combination (3.16), and the physical state at general level $2 n$ corresponds to the integral of the Ricci scalar to the $n^{\text {th }}$ power, i.e

$$
\left|2 n, p_{n}\right\rangle \rightarrow \int d^{4} x \sqrt{-g} R^{n}|\Omega\rangle
$$

as $Q^{2} \rightarrow \infty$.

Notice that for $n=1$ this operator becomes just the integral of the Ricci scalar in the large $Q^{2}$ limit. Moreover, for finite $Q^{2}$ the equation for the weight $\beta_{1}$ in (4.12) is identical to the equation for the scaling dimension of the conformal factor which sets the weight of the Ricci scalar density equal to 4 ,

$$
\left.w(\sqrt{-g} R)\right|_{g=e^{2 \alpha \sigma} \bar{g}}=2 \alpha-\frac{2 \alpha^{2}}{Q^{2}}+2=4
$$

obtained by the covariant methods of [1], with the identification $\beta_{1}=2 \alpha$. This operator mixes with the pure exponential $\lambda \exp (4 \alpha \sigma)$ in a well-defined way, the relative coefficient $\lambda$ determined by eq. (3.17) of ref. [1] and vanishing in the $Q^{2} \rightarrow \infty$ classical limit. This mixing phenomenon at finite $Q^{2}$ is an example of the operator mixing mentioned above which should generalize to higher levels for the operators $\sqrt{-g} R^{n}$.

The pure exponential operator found at level zero (4.5) with $\beta_{0}$ determined by (4.6) is identical to the volume operator added to the $\sigma$ action in ref. [11]. This operator and all of the higher level operators when added to the action (2.1) correspond to conformal deformations of the original "free" $\sigma$ theory, as encountered in two dimensional gravity with matter. It is characteristic of the non-trivial dynamics of the conformal factor in $D=4$ that there are such diffeomorphic invariant operators, even without the addition of any matter or transverse graviton fields.

Finally, one can repeat the physical state analysis for a conformal scalar field, $\Phi$, coupled to $\sigma$. The quantization and diffeomorphism generators for the scalar field are given in paper I. The energy-momentum tensor is now the sum of the contributions from the $\Phi$ and $\sigma$ fields. Starting with the Fock vacuum for the combined matter plus 
$\sigma$ theory, the general level $N$ state can be constructed by acting with the creation operators $\varphi^{\dagger}, a^{\dagger}, b^{\dagger}$. Applying first the volume non-preserving diffeomorphism constraints $\left(\dot{T}_{J M}^{(+)}[\Phi]+\dot{T}_{J M}^{(+)}[\sigma]\right)|p h y s\rangle=0$ leaves two kinds of states,

$$
\begin{aligned}
& \left(2(Q-i p) b_{00}^{\dagger}+\sum_{M} \epsilon_{M} a_{\frac{1}{2} M}^{\dagger} a_{\frac{1}{2}-M}^{\dagger}\right)^{n}\left(\varphi_{00}^{\dagger}\right)^{l}|0, p\rangle \\
& \text { and } \quad\left(\sqrt{2}(Q-i p) \varphi_{\frac{1}{2} M}^{\dagger}-a_{\frac{1}{2} M}^{\dagger} \varphi_{00}^{\dagger}\right)\left(\varphi_{00}^{\dagger}\right)^{l}|0, p\rangle .
\end{aligned}
$$

However the second class of states are not rotationally invariant (as is obvious from the free magnetic index $M$ ) and are eliminated by the global rotation constraint $R_{M_{1} M_{2}}|p h y s\rangle=0$. Hence the surviving physical states are a direct product of the states found in either the $\Phi$ or $\sigma$ theories separately. The Hamiltonian constraint at level $N=2 n+l$ determines the momentum eigenvalue $p_{N}$ by

$$
\frac{1}{2} p_{2 n, l}^{2}+\frac{1}{2} Q^{2}+2 n+l=4
$$

Clearly, in the classical limit $Q^{2} \rightarrow \infty$ the operators which create these physical states are of the form,

$$
\int d^{4} x \sqrt{-g} R^{n} \Phi^{l}|\Omega\rangle
$$

This example shows that the results obtained for the physical states and operators in the $\sigma$ theory are easily generalized in the presence of matter, at least in this limit. 


\section{Summary and Discussion}

In paper I we studied the constraints of coordinate invariance for any quantum theory possessing conformal symmetry independently of its particular dynamics. The DiracFock method of imposing the constraints requires only the vanishing of the matrix elements of the generators of spatial diffeomorphisms $\left\langle T^{0 i}\right\rangle$, because of their non-trivial commutators. We argued from the structure of the quantum algebra off-shell that the time reparametrization constraint generated by $T^{00}$ should not be imposed a priori, but only the weaker condition $\left\langle\dot{T}^{00}\right\rangle=0$. We found also that the Hamiltonian which is the spatial integral of $T^{00}$ is modified by a finite and calculable shift of -4 .

In this paper we applied this general formalism to the quantum theory of the conformal factor generated by the trace anomaly in four dimensions. Despite the fourth order effective action of this theory we found that the constraints of invariance under spatial diffeomorphisms eliminate all negative norm states. In the region of $Q^{2}<0$ normalizable states with real momentum exist and there is no violation of unitarity. In the region $Q^{2}>8$ which we regard as the physical region the physical states are non-normalizable and quite analogous to the discrete states of $2 \mathrm{D}$ quantum gravity in the region $c_{m} \leq 1$. The states are created by invariant scalar operators which in the classical limit $Q^{2} \rightarrow \infty$ correspond to integer powers of the scalar curvature integrated over spacetime. The scaling dimensions of these operators in the canonical approach provide an independent confirmation of the covariant treatment initiated in ref. [1]. Hence, the $\sigma$ effective action passes all the tests of a sensible quantum theory.

In a sense it is not surprising that the constraints of diffeomorphism invariance eliminate the negative norm states since this occurs even in the linearized Einstein theory. What is more remarkable is that there are any non-trivial states at all which survive in the complete absence of transverse gravitons. This confirms that the conformal factor is indeed dynamical (though non-propagating) in the full quantum theory, 
quite independently of the graviton degrees of freedom. Moreover, the physical states in this theory are created by operators possessing clear geometric significance in terms of the scalar curvature which is completely fixed by the equations of motion of the Einstein theory, but which fluctuates in the quantum theory. This is in contrast to two dimensions where the only effect of the Liouville mode is the gravitational dressing of matter operators.

In additional to some technical problems such as a more precise characterization of the operator mixing problem at finite $Q^{2}$, several issues remain to be addressed. Among these is the inclusion of transverse graviton modes, which although not expected to change the framework drastically should be investigated carefully. Another open problem is the correct characterization of the full algebra of quantum diffeomorphisms off-shell, which seems to be required for invariance under finite diffeomorphisms. If such a general algebra does not exist there may be serious implications for the consistency of the theory and/or for the Dirac-Fock approach to quantization which we have followed. Finally, the calculation of correlation functions in the $\sigma$ theory should provide non-trivial information about the far infrared behavior of quantum gravity in four dimensions with observable consequences in cosmology and the large scale structure of th Universe.

\section{Acknowledgments}

I.A. and P.O.M. would like to acknowledge the hospitality of Theoretical Division (T-8) of Los Alamos National Laboratory. E.M. and P.O.M. would like to thank the Centre de Physique Théorique of the Ecole Polytechnique for its hospitality. All three authors wish to acknowledge NATO grant CRG 900636 for partial financial support. 


\section{REFERENCES}

[1] I. Antoniadis and E. Mottola, Phys. Rev. D45 (1992) 2013;

I. Antoniadis, P. O. Mazur and E. Mottola, Phys. Lett. B323 (1994) 284.

[2] I. Antoniadis, P. O. Mazur, and E. Mottola, Nucl. Phys. B 388 (1992) 627.

[3] A. M. Polyakov, Phys. Lett. B103 (1981) 207, 211; Gauge Fields and Strings, (Harwood Academic, Chur, 1987).

[4] B. Allen and G. Turyn, Nucl. Phys. B292 (1987) 813;

E. G. Floratos, J. Iliopoulos and T. N. Tomaras, Phys. Lett. B197 (1987) 373;

I. Antoniadis and E. Mottola, Jour. Math. Phys. 32 (1991) 1037.

[5] V. G. Knizhnik, A. M. Polyakov, and A. B. Zamolodchikov, Mod. Phys. Lett. A3 (1988) 819.

[6] J. W. York, Phys. Rev. Lett. 26 (1971) 1656; ibid. 28 (1972) 1082;

Jour. Math. Phys. 13 (1972) 125; ibid. 14 (1973) 125.

[7] I. Antoniadis, P. O. Mazur and E. Mottola, Ecole Polytechnique preprint CPTH-S376.0995, and Los Alamos preprint LA-UR-95-3392, referred to as paper I in this work.

[8] T. L. Curtright and C. B. Thorn, Phys. Rev. Lett. 48 (1982) 1309;

Erratum, ibid. 1768;

J. L. Gervais and A. Neveu, Nucl. Phys. B199 (1982) 59;

B209 (1982) 125; B224 (1983) 329.

[9] L. S. Brown and J. P. Cassidy, Phys. Rev. D16 (1977) 1712.

[10] M. B. Green, J. H. Schwarz, and E. Witten, Superstring Theory, Vol. 1, (Cambridge Univ. Press, Cambridge, 1987).

[11] C. Schmidhuber, Nucl. Phys. B390 (1993) 188. 